\title{
'You can't have a mission without a camera'
}

\section{A sister of the Mars Express probe has made it to Venus. And scientists have travelled to Beijing to discuss its first results. Horst Uwe Keller, who is presenting images from the craft's camera, spoke to Nature from the meeting.}

I work on the Venus Monitoring Camera (VMC). One of our main objectives is to study the highest clouds, around 70 kilometres above the planet's surface.

The VMC is the only instrument newly developed for this mission, the rest were reused from Mars Express and the comet mission Rosetta. They didn't have a camera, so I stepped in and said: "You can't have a mission without a camera." We built something very small, $1-2 \mathrm{~kg}$, to replace the tiny camera on Mars Express that had watched the Beagle 2 lander as it separated.

The VMC is four cameras, one for ultraviolet light, one for visible and two for infrared. The results so far are qualitative we haven't finished calibrating the instrument

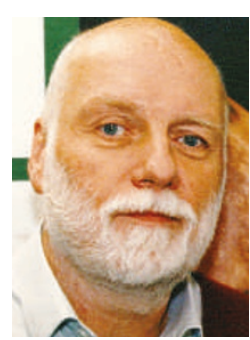

Planet photographer: Horst Uwe Keller. yet. But in the sequences of ultraviolet images I presented on Monday you can see the clouds moving, showing the wind direction.

As a young postdoc in the 1970s, I remember being at a meeting where a well-known astronomer claimed that Venus's clouds were made of droplets of sulphuric acid. At the time I thought he was crazy, but now we know this is true.

What we don't know yet is the nature of the 'ultraviolet absorber' that creates dark features in our cloud images. We hope one of the spectrometers will work out what it is.

We are also working on the infrared images, which we'll check for hot spots that might be active volcanism. The surface of Venus is very young, so we know there must be some volcan- ism, but no one has definitively seen it. If we're lucky we could also find out something about Venus's lightning using the visible-light filter.

Some of the most remarkable results at the meeting have come from VIRTIS - a spectrometer that probes the atmosphere from top to bottom. The pictures of the south pole are really spectacular: they show a double vortex created by the movement of the atmosphere between the equator and the pole.

For our team, however, the challenge is simply getting high-quality images. While the spacecraft was cruising into orbit, our camera ended up pointing at the Sun for about 50 hours. The Sun burnt its image into the photodetector. But it's not a complete disaster: we have been able to compensate for the damage by re-measuring the sensitivity of each pixel.

As told to Jenny Hogan. 